\title{
Green synthesis and antibacterial potential of artemisia vulgaris extract in silver nanoparticles against wound bacteria
}

\author{
Laura Soon ${ }^{*}$, Phui Qi Ng${ }^{1}$, \\ Jestin Chellian ${ }^{2}$, Thiagarajan Madheswaran ${ }^{3}$, Jithendra Panneerselvam ${ }^{3}$, Alan $\mathrm{Hsu}^{4}$, \\ Philip Michael Hansbro ${ }^{5,6,7}$, Kamal Dua ${ }^{5,8,9}$, Trudi Collet ${ }^{10}$, Dinesh Kumar Chellappan ${ }^{2}$
}

\begin{abstract}
${ }^{1}$ School of Pharmacy, International Medical University, 57000 Kuala Lumpur, Malaysia;
${ }^{2}$ Department of Life Sciences, International Medical University, 57000 Kuala Lumpur, Malaysia

${ }^{3}$ Department of Pharmaceutical Technology, International Medical University, 57000 Kuala Lumpur, Malaysia

${ }^{4}$ School of Medicine and Public Health, The University of Newcastle, NSW 2308, Australia;

${ }^{5}$ Centre for Inflammation, Centenary Institute, Sydney, NSW 2050, Australia;

${ }^{6}$ Priority Research Centre for Healthy Lungs, Hunter Medical Research Institute, New Lambton, NSW 2305

and The University of Newcastle, Callaghan, NSW 2208, Australia

${ }^{7}$ Faculty of Science, University of Technology Sydney, Ultimo NSW 2007, Australia;

${ }^{8}$ Discipline of Pharmacy, Graduate School of Health, University of Technology Sydney, Sydney, NSW 2007, Australia

${ }^{9}$ School of Biomedical Sciences and Pharmacy, University of Newcastle, NSW 2308, Australia

${ }^{10}$ Innovative Medicines Group, Institute of Health and Biomedical Innovation, Queensland University of Technology (QUT), Kelvin Grove, Brisbane, Queensland 4059, Australia
\end{abstract}

${ }^{*}$ Corresponding author : laura soon@hotmail.com

\begin{abstract}
Background: Artemisia vulgaris (A. vulgaris), a well-known Chinese traditional herb, is reported to have antibacterial properties, making it a potential agent for wound healing. In our project, we have developed A. vulgaris in silver nanoparticles to enhance its effect. This study investigated the antibacterial effects of the synthesised AgNP on common wound bacteria.

Objectives: The objectives of this study were to synthesise A. vulgaris in silver nanoparticles and to investigate the anti-bacterial effect on wound bacteria.

Methods: The AgNP was synthesised by the green synthesis method and characterisation tests were carried out to confirm the presence of AgNP in the formulation. The disc diffusion test, minimum inhibitory concentration (MIC), and minimum bactericidal concentration (MBC) tests were carried out to investigate the antibacterial effects of AgNP on common wound bacteria. The AgNP was also tested on probiotics using the disc diffusion test to investigate its effect on probiotics.

Results: The characterisation tests have confirmed the presence of AgNP in the formulation. The AgNP containing all plant concentrations were able to inhibit the growth of all bacteria tested but it required a higher concentration to inhibit the gram positive bacteria. The AgNP had less inhibitory effects on probiotics compared to antibiotics and silver nitrate alone. However, statistical analysis showed that the antibacterial effect of the treatment was statistically insignificant.

Conclusion: The AgNP demonstrated anti-bacterial effects on both gram positive and gram negative wound bacteria, but the effect of the treatment was not statistically significant.

Keywords: Artemisia vulgaris, silver nanoparticles, antibacterial, wound bacteria

\section{Introduction}

Wound infection affects cuts, burns, surgical wounds, and diabetic wounds when pathogens invade through the wound opening, resulting in poor wound healing, gangrene, disability, sepsis, and death (Efron \& Barbul, 2001). Normal skin flora may become pathogens that invade wound openings when given the right circumstances. Some of these bacteria have
\end{abstract}


developed resistance towards the standard antibiotic treatment (Stapleton \& Taylor 2007). Hence, the development of new antibacterial agents is important to overcome such resistance. Artemisia vulgaris, a Chinese traditional herb, is one of the plants which has a potential antibacterial effect. Commonly known as mugwort, it is often used as a traditional remedy or culinary herbs. It is much favoured by the Chinese because it is claimed to have numerous health benefits. It has produced positive results in previous antimicrobial and antioxidant studies (Temraz \& El-Tantaway, 2008; Manindra, M. et al, 2016) It is also traditionally used to treat various types of health ailments, such as bleeding, menstrual problems, and skin problems (Fetrow \& Avila, 2004).

Herbal agents such as $A$. vulgaris face challenges in terms of drug delivery and bioavailability due to their poor stability and rapid elimination. The extract of $A$. vulgaris can be incorporated into a novel delivery system such as silver nanoparticles to enhance its antibacterial effects while providing a safer alternative for the treatment of wound infection. Silver nanoparticles have been used as a carrier for many herbal agents and shown to have synergistic effects (Aparna, et al, 2015; Orsuwan, et al, 2017). Therefore, in this study, silver nanoparticles containing $A$. vulgaris extracts (AgNP) were tested on common wound bacteria to determine its effectiveness as an antibacterial agent.

\section{Methods}

\subsection{Chemicals and reagents}

Silver nitrate was obtained from ACROS Organics ${ }^{\mathrm{TM}}$ and used at a concentration of $0.1 \mathrm{M}$ to formulate silver nanoparticles. Mueller-Hinton Media was procured from Sigma-Aldrich.

\subsection{Preparation of plant extracts}

The leaves of A. vulgaris were collected from a private garden in Kota Kinabalu, Sabah. The herbarium specimen was then sent to the Sabah Forestry Department to be validated by the Forest Research Centre. Around 500g of leaves collected were dried and ground into coarse powder form. The coarse leaf powder was boiled in $100 \mathrm{~mL}$ of distilled water at $80^{\circ} \mathrm{C}$ for 3 hours to prepare the aqueous plant extracts in $5 \%, 10 \%$ and $15 \% \mathrm{w} / \mathrm{v}$ concentrations. The extracts were then filtered with common filter paper. (Ahmed, et al, 2016)

\subsection{Green synthesis of silver nanoparticles}

To synthesise the plant extract into silver nanoparticles, the filtered extracts were mixed with $50 \mathrm{mM}$ silver nitrate solution at a ratio of $1: 9 \mathrm{v} / \mathrm{v}$ (Erjaee, et al, 2017). The 50mM silver nitrate solution was prepared earlier by diluting $0.1 \mathrm{~N}$ Acros Organics ${ }^{\mathrm{TM}}$ Silver nitrate. The mixture of plant extract and silver nitrate was incubated at a room temperature under the stirring condition for 18 hours. The solution was then centrifuged at $13000 \mathrm{rpm}$ for 20 minutes 
to separate the nanoparticles from the solution. The nanoparticles obtained were washed with distilled water to remove any unwanted materials.

\subsection{Characterisation of silver nanoparticles}

The UV-visible absorption of the silver nanoparticles was determined in quartz cuvette using the Perkin Elmer spectrometer. The wavelength range was taken from 300 to $800 \mathrm{~nm}$. FTIR spectroscopy was obtained using the ATR method and conducted at a room temperature under dry air. The wave range was set to $4000-400 \mathrm{~cm}^{-1}$ (Uznanski, et al, 2017). The particle size of silver nanoparticles was analysed using the Malvern Zetasizer Nano Instrument to determine the particle size distribution and surface charge. A high-resolution transmission electron microscope (Hitachi HT 7700) was employed to analyse the surface morphology and size of silver nanoparticles.

\subsection{Investigation of antibacterial effects of AgNP on wound bacteria}

The antibacterial tests were performed on common wound bacteria, which included $K$. pneumonia, P. aeruginosa, E. coli, B. cereus, S. aureus, and two strains of MRSA.

\subsubsection{Disc diffusion test}

Muller Hinton Agar (MHA) medium was prepared and the bacterial culture of 0.5 McFarland standard was spread thoroughly on the agar plates. The silver nanoparticles containing $5 \%, 10 \%$ and $15 \% \mathrm{w} / \mathrm{v}$ of plant extract were made into a solution and added into the wells made on the agar plates. Antibiotic discs were used as the positive control whereas $50 \mathrm{mM}$ silver nitrate was used as the negative control. The plates were incubated overnight at $37^{\circ} \mathrm{C}$. The diameter of inhibition zone was indicative of the inhibitory effect of silver nanoparticles on the growth of the bacteria.

\subsubsection{Minimum Inhibitory Concentration (MIC) test}

The culture medium, bacterial suspension, and formulation samples with plant extract concentration ranging from $0.125 \mathrm{mg} / \mathrm{mL}$ to $4 \mathrm{mg} / \mathrm{mL}$ were added into a 96 -well plate and then incubated overnight at $37^{\circ} \mathrm{C}$. Dyes were added into each well to analyse the results. The MIC is the lowest concentration where bacterial growth is inhibited by $50 \%$.

\subsubsection{Minimum Bactericidal Concentration (MBC) test}

Using the samples from MIC test, $5 \mu \mathrm{l}$ of sample was taken from each well and added onto the agar plate. The plates were then incubated at $37^{\circ} \mathrm{C}$ overnight. The lowest concentration with no bacterial growth observed on the plates was considered the MBC. 


\subsection{Investigation of antibacterial effects of AgNP on probiotics}

The disc diffusion test was carried out on probiotics, namely L. casei, L. rhamnosus, $L$. arabinosus, and L. acidophilus. The procedure was the same as that carried out on wound bacteria.

\subsection{Statistical analysis}

The Optima Data Analysis software version 2 was used to analyse the data obtained from the antibacterial tests of AgNP. ANOVA test was used to compare variables between groups. Statistical significance was set to $<0.05$ for all tests.

\section{Results and Discussion}

\subsection{Characterization of silver nanoparticles}

In accordance to previous studies, a reduction reaction of silver nitrate to silver takes place when plant extracts are added (Sathishkumar, et al, 2009; Rasheed, et al, 2017). The reaction can also be confirmed by the UV spectrum, where a broad absorption peak can be seen with $\lambda \max$ at $427 \mathrm{~nm}$, indicating the presence of silver nanoparticles (Figure 1). The plant extract itself is capable of reducing silver nitrate without the use of synthetic chemical reagents.

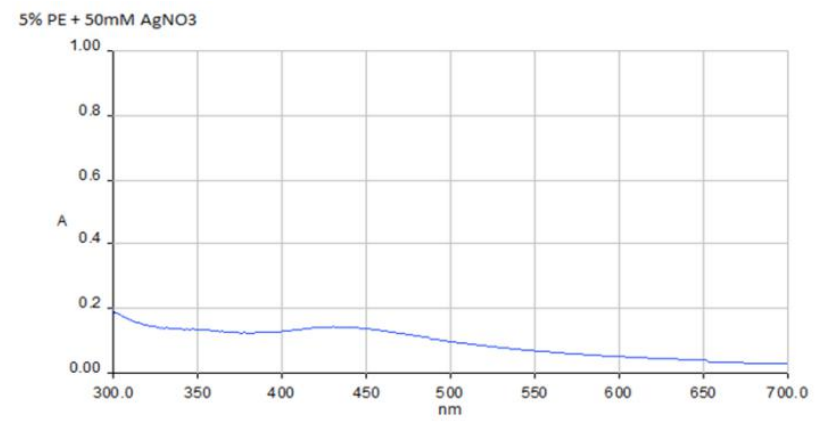

Figure 1: The UV spectra obtained which indicate the presence of silver nanoparticles

From the FTIR spectrum, significant absorption peaks of around $1100 \mathrm{~cm}$ and $1300 \mathrm{~cm}$ can be observed (Figure 2) The bands at $1100 \mathrm{~cm}$ and $1300 \mathrm{~cm}$ indicate the stretching of alkyl amine and alkyl ketone respectively. These functional groups present in A. vulgaris extracts were responsible for such reactions.

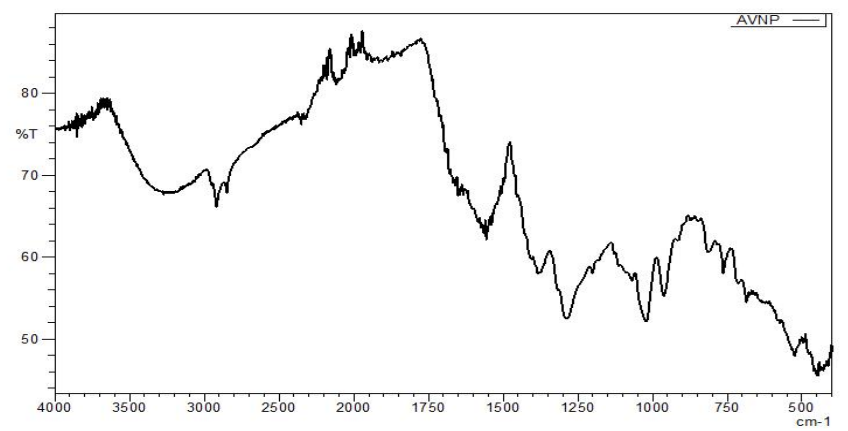

Figure 2: The FTIR spectra showing the functional groups responsible for the reduction reaction to produce silver nanoparticles 
The data obtained using zetasizer show that the z-average for the nanoparticles containing $5 \%, 10 \%$, and $15 \%$ plant extracts were $123 \mathrm{~nm}, 240 \mathrm{~nm}$, and $237 \mathrm{~nm}$, respectively. The particle size estimated by zetasizer seemed to be larger than the usual nanoparticle size. Although the particle size was estimated to be around $200 \mathrm{~nm}$, the morphological analysis using TEM showed that most particle sizes fell within $50 \mathrm{~nm}$. The zeta potential ranged $+20-30 \mathrm{mV}$ for all concentrations, indicating that the formulation was stable (Table 1).

Table 1: Particle size and zeta potential of silver nanoparticles containing plant extract

\begin{tabular}{ccc}
\hline $\begin{array}{c}\text { Concentration of plant } \\
\text { extract in AgNP }\end{array}$ & $\begin{array}{c}\text { Mean particle size } \\
\text { (mm) }\end{array}$ & Zeta Potential (mV) \\
\hline $50 \mathrm{mg} / \mathrm{mL}$ & 152.3 & +24.6 \\
$100 \mathrm{mg} / \mathrm{mL}$ & 224.5 & +29.0 \\
$150 \mathrm{mg} / \mathrm{mL}$ & 264.4 & +30.1 \\
\hline
\end{tabular}

The morphological analysis using TEM shows that the nanoparticles displayed a globular shape with a size ranging from 20 to $50 \mathrm{~nm}$ (Figure 3). The aggregation of silver nanoparticles can be observed, which explains the larger size estimation by zetasizer. It is common that silver nanoparticles aggregate to be in a stable form (Prathna, 2011).

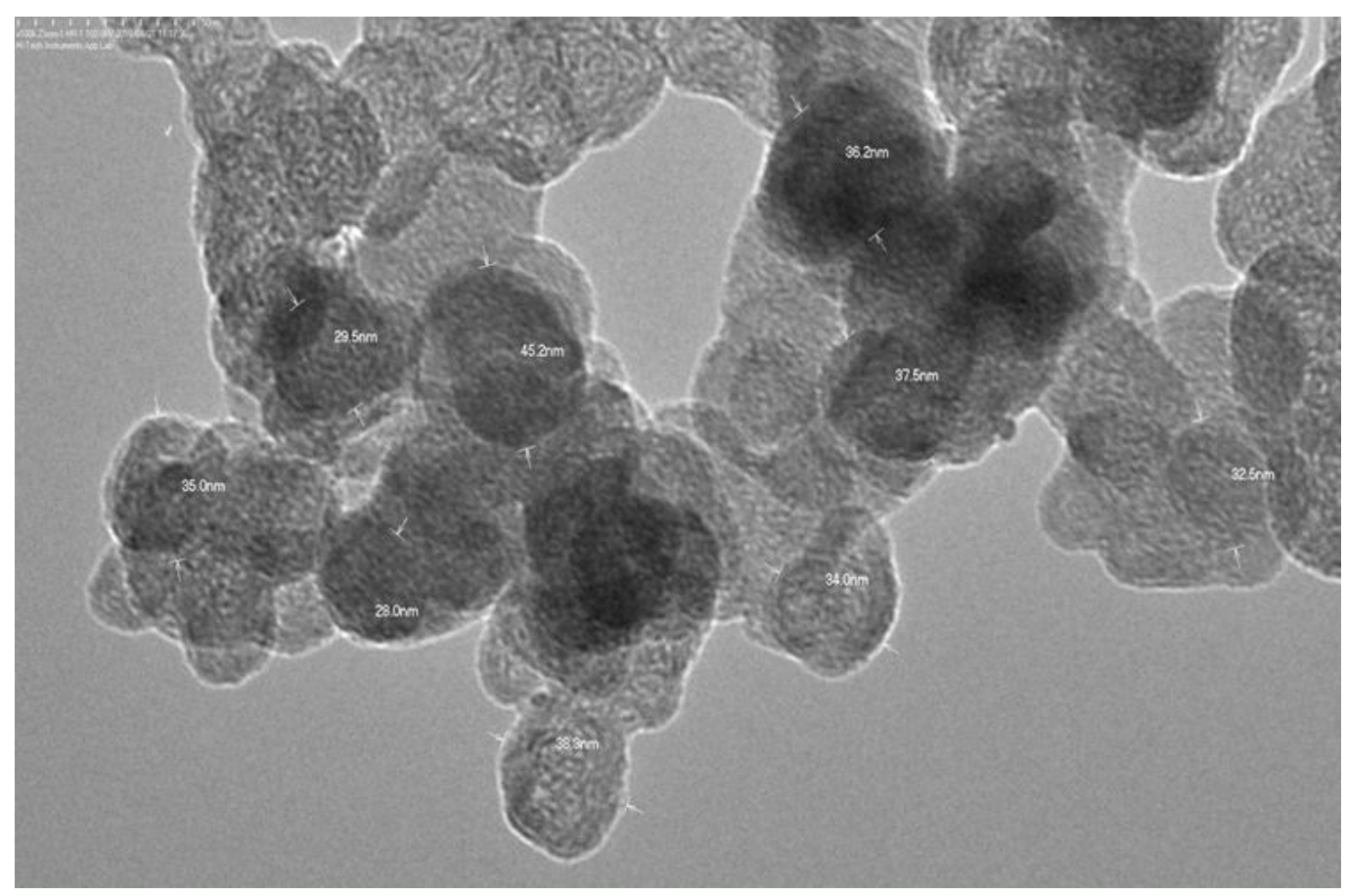

Figure 3: TEM image at 50nm magnification showing the silver nanoparticles with the size ranging between 20 and $50 \mathrm{~nm}$

\subsection{Investigation of antibacterial effects of AgNP on wound bacteria}

\subsubsection{Disc diffusion plate test}

AgNP with all concentrations of plant extract was able to inhibit the growth of gram positive and gram negative bacteria, although the antibacterial effect was not significant. 
(Figure 4). The combination was better than both $50 \mathrm{mM}$ silver nitrate alone and plant extract alone. However, the AgNP was still not as effective as the positive control, the antibiotic discs. The bacterial growth inhibition was not affected by the concentration of plant extracts.

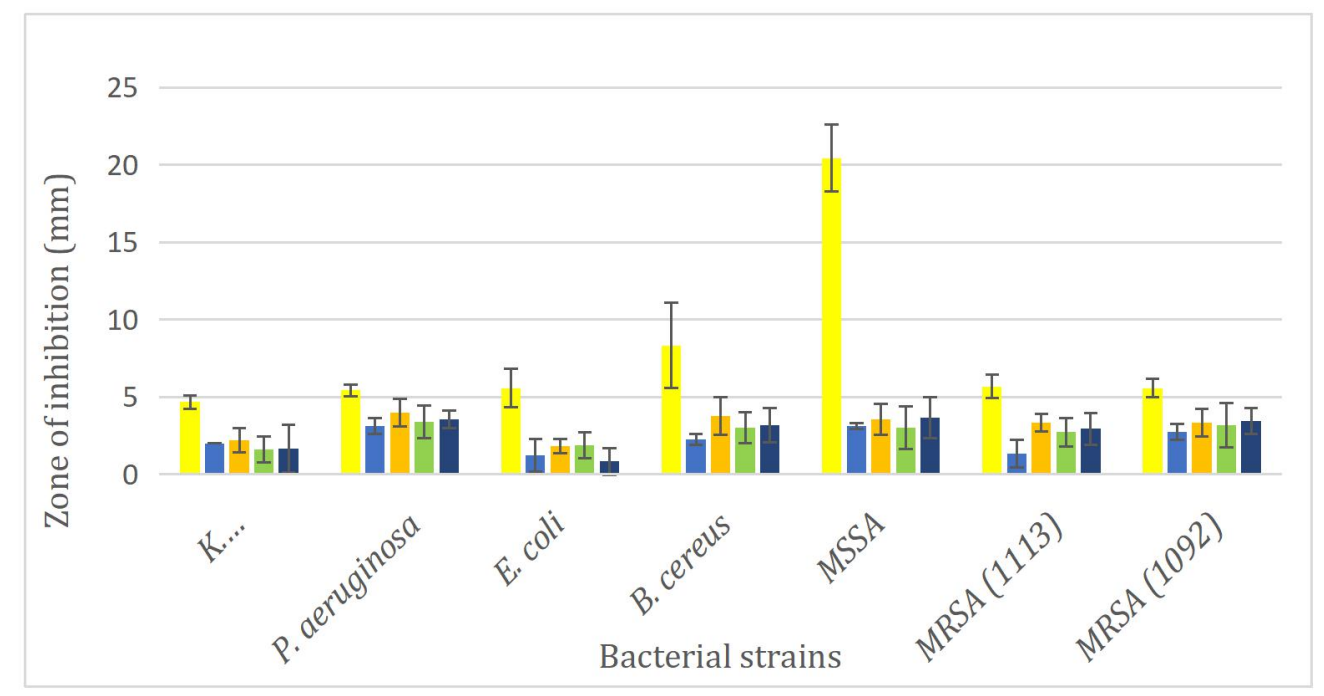

Figure 4: The inhibition of bacterial growth by disc diffusion plate test

The results indicated that the silver nanoparticles and A. vulgaris extracts can enhance each other's effect to inhibit the growth of bacteria. According to previous studies, the antibacterial effect of silver nanoparticles is mainly attributed to its small size and electrostatic attraction. (Rasheed, et al, 2017; Prathna, 2011; Nam, et al, 2015). The small size of silver nanoparticles allows it to easily penetrate the bacterial membrane. The small size of nanoparticles provides a high-surface-to-volume ratio, allowing them to have an increased contact area on the bacterial surface so that a greater amount of silver ions can exert the bactericidal effects towards the bacteria (Nam, et al, 2015; Bondarenko, et al, 2013).

The positively-charged nanoparticles and negatively-charged cell surface of gram negative bacteria cause an electrostatic attraction, which eases the diffusion of nanoparticles into bacterial cells (Pazos-Ortiz, E., et al, 2017). The permeation of nanoparticles into bacteria may result in the disruption of protein synthesis, alteration of bacterial structure, and cell death. The plant extract of A. vulgaris has a minimal bacterial effect compared to silver nitrate alone. The methanolic extract of A. vulgaris is slightly better than the aqueous extract. Terpene compounds found in A. vulgaris may contribute to its antibacterial effect (Zengin \& Baysal, 2014)

\subsubsection{Minimum Inhibitory Concentration (MIC)}

From the MIC values, the silver nanoparticles containing $A$. vulgaris extract were effective in inhibiting both the gram positive and gram negative bacteria (Figure 5). The gram negative bacteria, E. coli and K. pneumonia, had the lowest MIC value, $0.25 \mathrm{mg} / \mathrm{mL}$. Both methicillin susceptible and resistant strains of S. aureus had the highest MIC value of $1.00 \mathrm{mg} / \mathrm{mL}$. 


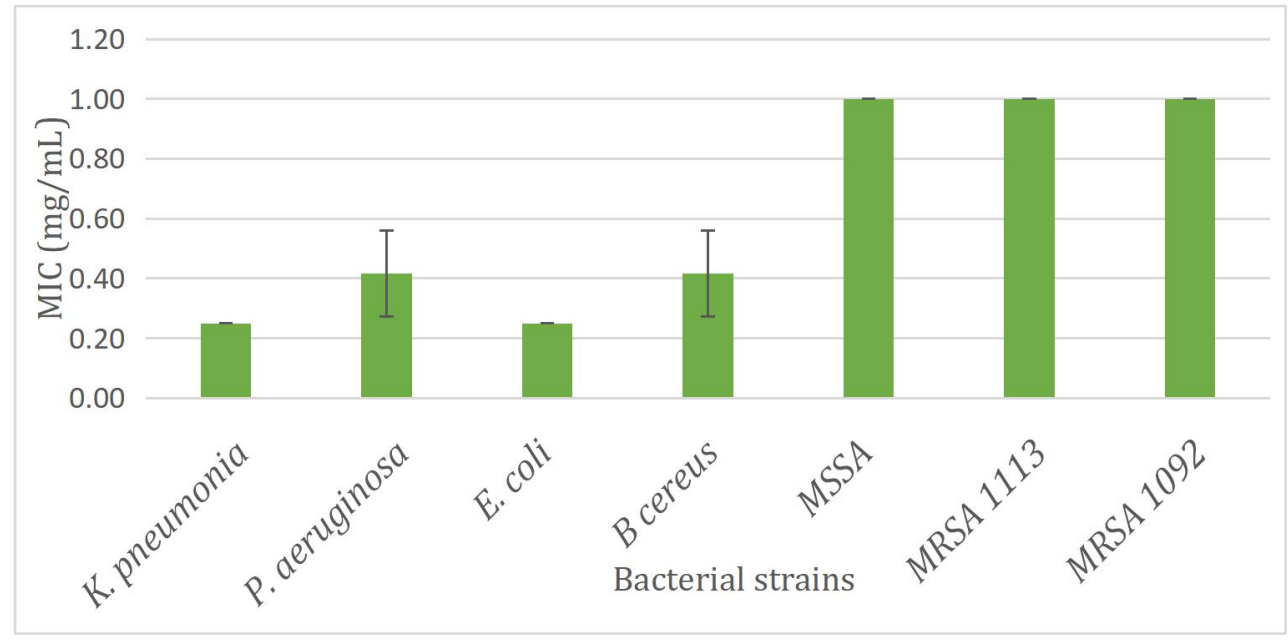

Figure 5: The minimum concentration required to inhibit bacterial growth by $50 \%$

Although silver nanoparticles containing A. vulgaris extract were effective in inhibiting the growth of gram positive and gram negative bacteria, the results suggested that the inhibition towards gram negative bacteria was more prominent than that on gram positive ones. From the MIC values, it can be observed that a higher concentration of the formulation was required to inhibit the growth of gram positive bacteria compared to gram negative bacteria. The thick cell wall of gram positive bacteria contains a higher amount of peptidoglycan which causes the silver ions to adhere on the cell wall, resulting in a poorer antibacterial effect (Dakal, et $\mathrm{al}$, 2016). The cell membrane of gram negative bacteria possesses lipopolysaccharides which are negatively charged. This promotes the adhesion of silver nanoparticles, causing the bacteria to be more susceptible to the antibacterial effect (Dakal, et al, 2016). The mechanism of A. vulgaris extract between gram negative and gram positive bacteria, however, is still not fully understood.

\subsubsection{Minimum Bactericidal Concentration (MBC)}

From the MBC values, it is shown that $4 \mathrm{mg} / \mathrm{mL}$ of silver nanoparticles containing $\mathrm{A}$. vulgaris extract, which was the highest concentration, was unable to kill the bacterial population of all the strains tested.

Table 2: Results of the MBC test

\begin{tabular}{cc}
\hline Bacteria & Minimum Bactericidal Concentration $(\mathbf{m g} / \mathbf{m L})$ \\
\hline K. pneumonia & $\sim 4$ \\
P. aeruginosa & $>4$ \\
E. coli & $>4$ \\
B cereus & $>4$ \\
MSSA & $>4$ \\
\hline
\end{tabular}




\subsection{Investigation of antibacterial effects of AgNP on probiotics}

AgNP demonstrated lower antibacterial effects towards probiotics when compared to standard antibiotics (Figure 6). It is also noted that plant extract alone had less inhibition towards the growth of probiotics compared to AgNP. This suggests that the plant extracts exhibit a protective effect towards good bacteria so that the cells can be protected from the damaging effects of silver nanoparticles.

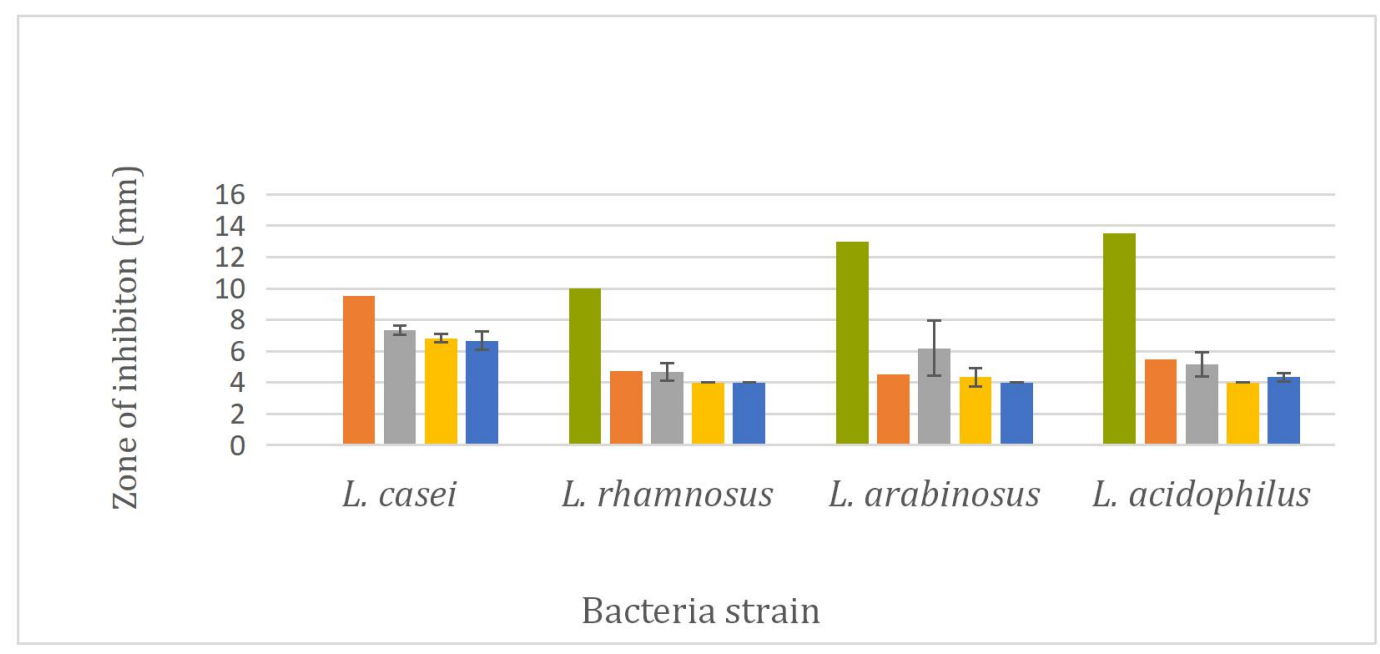

Figure 6: The inhibition of probiotics growth by disc diffusion plate test

A study has shown that a diet containing A. vulgaris was linked to an increase in intestinal bifidobacteria (Lee, et al, 1995). In a case report, $A$. vulgaris has been shown to speed up the wound healing process of an anaconda snake, indicating skin protective effects. In previous studies, $A$. vulgaris has shown cell protective effects, including hepatoprotective effects and less cytotoxic effects towards normal cells when compared to cancer cells (Gilani, et al, 2005; Saleh, et al, 2014)

\section{Conclusion}

A. vulgaris extracts and silver nanoparticles enhance each other's effect to inhibit the growth of bacteria, although not significantly. The plant extracts also exhibit a protective effect, protecting the probiotics from the damaging effects of treatment. Hence, silver nanoparticles containing A. vulgaris extract are a potentially safer alternative to the standard antibacterial treatment.

\section{Acknowledgment}

The research was carried out in collaboration with Queensland University of Technology, Australia, and supported by a grant from the International Medical University (IMU), Malaysia (Project ID: BP I-01/2018(39)). 


\section{References}

Ahmed. S., Ahmad, M., Swami, B.L., \& Ikram, S. (2016). A review on plants extract mediated synthesis of silver nanoparticles for antimicrobial applications: a green expertise. J $A d v$ Res, 7(1), 17-28.

Aparna Mani, K.M., Seethalakshmi, S. \& Gopal, V. (2015). Evaluation of in-vitro antiInflammatory activity of silver nanoparticles synthesised using Piper nigrum extract. $J$ Nanomed Nanotechnol, 6(2), doi: 10.4172/2157-7439.1000268.

Bondarenko, O., Ivask, A., Käkinen, A., Kurvet, I., \& Kahru, A. (2013). Particle-cell contact enhances antibacterial activity of silver nanoparticles. PLoS ONE. 2013; 8: e64060.

Efron, D.T. \& Barbul A. (2001). Wounds in infection and sepsis - role of growth factors and mediators. In: Holzheimer RG, Mannick JA, editors. Surgical Treatment: Evidence-Based and Problem-Oriented. Munich: Zuckschwerdt; 2001. Available from: https://www.ncbi.nlm.nih.gov/books/NBK6957/.

Erjaee, H., Rajaian, H., \& Nazifi, S. (2017). Synthesis and characterization of novel silver nanoparticles using chamaemelum nobile extract for antibacterial application. Adv Nat Sci: Nanosci Nanotechnol, 8, 1-9.

Dakal, T.C., Kumar, A., Majumdar, R.S., \& Yadav, V. (2016). Mechanistic Basis of Antimicrobial Actions of Silver Nanoparticles. Front Microbiol. doi:10.3389/fmicb.2016.01831.

Fetrow, C. \& Avila, J. (2004). Professional's Handbook of Complementary \& Alternative Medicines. Philadelphia, PA: Lippincott Williams \& Wilkins.

Gilani, A.H., Yaeesh, S., Jamal, Q., \& Ghayur, M.N. (2005). Hepatoprotective activity of aqueousmethanol extract of Artemisia vulgaris. Phytother Res, 19(2), 170-172.

Lee, S. H., S. J. Woo, Y. J. Koo \& H. K. Shin. (1995). Effects of mugwort, onion and polygalae radix on the intestinal environment of rats. Korean J. Food Sci. Technol. 27, 598-604.

Manindra M, Pandey AK, Nautiyal MK, \& Singh P. (2016). Antioxidant and antimicrobial activities of three Artemisia vulgaris (L.) essential oils from Uttarakhand, India. Journal of Biologically Active Products from Nature, 6(3), 266-271.

Nam, G., Rangasamy, S., Purushothaman, B. \& Song, J.M. (2015). The application of batericidal silver nanoparticles in wound treatment. Nanomater Nanotechnol. 5(23).

Orsuwan, A., Shankar, S., Wang, L.F., Sothornvit, R., \& Rhim, J.W. (2017). One-step preparation of banana powder/silver nanoparticles composite films. J Food Sci Technol, 54(2), 497-506.

Pazos-Ortiz, E., et al. (2017) Dose-dependent antimicrobial activity of silver nanoparticles on polycaprolactone fibers against Gram-positive and Gram-negative bacteria. J Nanomater, doi.org/10.1155/2017/4752314.

Prathna, T.C., Chandrasekaran, N., Mukherjee, A. (2011). Studies on aggregation behaviour of silver nanoparticles in aqueous matrices: Effect of surface functionalization and matrix composition. Colloids and Surfaces A: Physiochemical and Engineering Aspects, 390 (1-3), 216-224.

Rasheed, T., Bilal, M., Iqbal, H.M.N., \& Li, C. (2017). Green biosynthesis of silver nanoparticles using leaves extract of Artemisia vulgaris and their potential biomedical applications. Colloiods Surf B Biointerfaces, 158, 408-415.

Saleh, A.M., Aljada, A., Rizvi, S.A.A., Nasr, A., Alaskar, A.S., \& Williams, J.D. (2014). In vitro cytotoxicity of Artemisia vulgaris L. essential oil is mediated by a mitochondriadependent apoptosis in HL-60 leukemic cell line. BMC Complement Altern Med, 14(1), 115.

Sathiskumar, M., Sneha, K., \& Won, S.W. (2009). Cinnamon zeylanicum bark extract and powder mediated green synthesis of nano-crystalline siver particles and its bactericidal activity. Colloids Surf B Biointerfaces, 73(2), 332-338.

Stapleton, P.D. \& Taylor P.W. (2007). Europe PMC Funders Group methicillin resistance in Staphylococcus aureus : methicillin resistance. Sci Prog, 85, 1-14.

Temraz A., \& El-Tantaway, W.H. (2008) Characterisation of antioxidant activity of extract from Artemisia vulgaris. PakJ Pharm Sci, 21(4), 321-6. 
Uznanski, P., Zakrzewska, J., Favier, F., Kazmierski, S., \& Bryszewska, E. (2017) Synthesis and characterization of silver nanoparticles from (bis)alkylamine silver carboxylate precursors. J Nanopart Res, 19(3), 121.

Zengin, H., \& Baysal, A.H. (2014). Antibacterial and antioxidant activity of essential oil terpenes against pathogenic and spoilage-forming bacteria and cell structure-activity relationships evaluated by SEM microscopy. Molecules, 19(11), 17773-98. 